\title{
Acute triiodothyronine treatment and red blood cell sedimentation rate (ESR) in critically ill COVID-19 patients: A novel association?
}

Constantinos Pantos ${ }^{\mathrm{a}, *}$, Vassiliki Apostolaki ${ }^{\mathrm{b}}$, Leonidas Kokkinos ${ }^{\mathrm{b}}$, Athanassios Trikas ${ }^{\mathrm{a}}$ and Iordanis Mourouzis ${ }^{\mathrm{a}}$

${ }^{a}$ Department of Pharmacology, Faculty of Medicine, National and Kapodistrian University of Athens, Athens, Greece

${ }^{\mathrm{b}}$ Department of Anesthesiology, ELPIS General Hospital of Athens, Athens, Greece

\begin{abstract}
Sepsis and septic shock result in impaired microcirculation and red blood cell rheology which lead to tissue hypoxia and multi-organ failure. Early administration of triiodothyronine prevents tissue hypoxia in experimental sepsis. In this context, a clinical trial was initiated to test the efficacy of acute triiodothyronine administration to combat tissue hypoxia in critically ill COVID19 patients. Here, we provide preliminary data from interim analysis of this study showing a novel acute effect of triiodothyronine on erythrocyte sedimentation rate which may have an important therapeutic impact on red blood cell rheology and tissue hypoxia in sepsis and particular in COVID19 critical illness.

Trial registration: ClinicalTrials.gov, NCT04348513. Registered 16 April 2020, https://clinicaltrials.gov/ct2/show/ NCT04348513
\end{abstract}

Keywords: Thyroid hormone, hypoxia, erythrocyte sedimentation rate, COVID19, microcirculation, hemorheology

\section{List of abbreviations}

$\begin{array}{ll}\text { COVID } & \text { Coronovirus Disease } \\ \text { ESR } & \text { Erythrocyte sedimentation rate } \\ \text { HIF- } \alpha & \text { Hypoxia Inducible Factor }-\alpha \\ \text { HIV } & \text { Human Immunodeficiency Virus } \\ \text { p38 MAPK } & \text { p38 Mitogen-Activated Protein Kinase } \\ \text { T3 } & \text { Triiodothyronine } \\ \text { T4 } & \text { Thyroxine } \\ \text { TSH } & \text { Thyroid Stimulating Hormone }\end{array}$

Impairment of microcirculation remains one of the main pathophysiological mechanisms of tissue hypoxia in sepsis and septic shock. Tissue hypoxia leads to cell apoptosis and tissue injury and to neovessel formation with abnormal vasomotor response, increased vascular permeability and thrombosis

\footnotetext{
${ }^{*}$ Corresponding author: Constantinos Pantos, Micras Asias 75, 11527 Athens, Greece. Tel.: +30 210 746 2560; E-mail: cpantos@med.uoa.gr.
} 

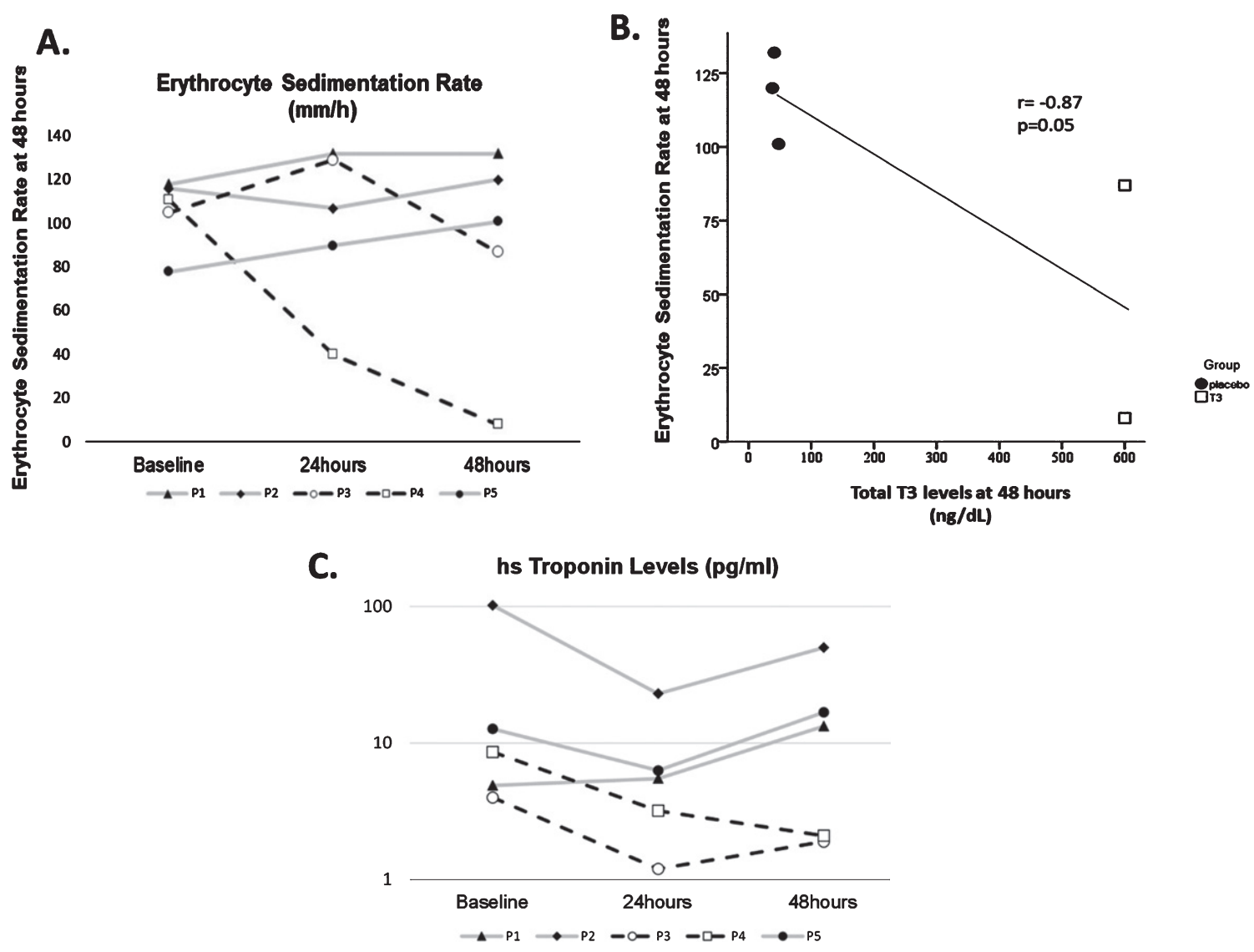

Fig. 1. A. Levels of erythrocyte sedimentation rate for each patient at baseline, 24 hours and 48 hours. Patients P3 and P4 received high dose T3 treatment (black line), while $\mathrm{P} 1, \mathrm{P} 2$ and $\mathrm{P} 5$ received placebo (grey line). $P=$ patient. B. Correlation of erythrocyte sedimentation rate with total T3 levels at 48 hours. C. Levels of highly sensitive Troponin for each patient at baseline, 24 hours and 48 hours. Patients P3 and P4 received high dose T3 treatment (black line), while P1, P2 and P5 received placebo (grey line). $P=$ patient.

(pathologic angiogenesis) phenomena which further worsen hypoxia (hypoxia vicious circle) and result in multi-organ failure. At present, there are no available effective treatments to prevent tissue hypoxia in sepsis.

Recent research provides evidence that administration of triiodothyronine (T3) can prevent tissue hypoxia in experimental sepsis and significantly reduce circulating lactate levels. Interestingly, T3 prevented the reduction of tissue oxygen levels below the threshold that triggers HIF- $\alpha$ dependent mechanisms which promote pathological angiogenesis [1]. In addition, T3 can prevent ischemia/reperfusion induced apoptosis via regulation of the pro-apoptotic p38 MAPK and pro-survival Akt signaling pathways [2].

In this context, the potential use of triiodothyronine treatment for combating tissue hypoxia is under investigation in COVID-19 critically ill patients in a randomized, double-blind, placebo-controlled study (ThySupport, NCT04348513, EudraCT:2020-001623-13) [3]. The first early interim analysis to assess the acute effects of triiodothyronine treatment revealed a novel association of T3 levels and erythrocyte sedimentation rate (ESR) which may be of important physiological and therapeutic relevance. This analysis included 5 consecutive patients intubated and treated according to study protocol [4] with high dose $\mathrm{T} 3$ for $48 \mathrm{~h}$ (two patients) and placebo (3 patients). The age (mean $\pm \mathrm{SD}$ ) of 
patients was $65.2 \pm 7.5$ years. All patients had severe pneumonia with mean of $\mathrm{pO}_{2} / \mathrm{FiO}_{2}=121.6 \pm 17.7$ on admission to Intensive Care Unit. Circulating total T3 levels ( $44 \pm 6.5 \mathrm{ng} / \mathrm{dL}$ ) before initiation of treatment were significantly suppressed as compared to normal range levels $(64-152 \mathrm{ng} / \mathrm{dL}$ for total T3). All patients were on dexamethasone treatment. Administration of triiodothyronine was associated with an acute drop in ESR (Fig. 1A). Furthermore, a strong inverse correlation between circulating T3 and ESR at 48 hours was observed (Fig. 1B).

ESR remains a common clinical tool to assess the acute phase response and disease progression and is an indirect index of red blood cell aggregation and altered red blood cell rheology [5]. In addition, it has been associated with inflammatory and vascular endothelial function indices [6]. In the context of COVID-19, changes in erythrocyte function and microcirculation have been considered as an important part of the pathophysiology of the disease and need to be deeply investigated in order new effective treatments to emerge [7-9]. Along this line, red blood cell rheological properties are found to be impaired in COVID-19 [10,11].

Taken together, our preliminary observations indicate a novel action of triiodothyronine on red cell blood aggregation with potential impact on red blood rheology, tissue hypoxia and organ function in COVID-19 critically ill patients. Indeed, our preliminary analysis showed a trend for lower troponin levels in patients receiving T3 treatment. Figure 1C. The small sample of patients used in this preliminary analysis does not allow any conclusions to be drawn on potential clinical impact of triiodothyronine treatment on patients' outcome. This issue remains to be further addressed. However, these preliminary clinical observations may shed some light on the potential mechanisms which are involved in the triiodothyronine novel effect on preventing tissue hypoxia as this was observed in experimental sepsis [1]. Therapeutic targeting of microcirculation and tissue hypoxia remains an unmet need in sepsis and particularly in COVID-19 in which hypoxia prevails $[8,12]$.

\section{Declarations}

Ethics approval and consent to participate

Thy-Support study (NCT04348513, EudraCT:2020-001623-13). The study protocol and any amendments were reviewed and approved by the National Independent Ethics Committee (45309/2020, 21-5-2020) and the Greek Drug Agency (45284/47235/49287, 8-5-2016 and IS code 31/20). The trial was performed in accordance with the Declaration of Helsinki (revised version, 1996), the European Guidelines for Good Clinical Practice (version 11, July 1990).

\section{Consent for publication}

The work described has not been published before and is not under consideration for publication anywhere else. The submitted work is original and has been approved for publication by all co-authors.

\section{Availability of data and material}

The datasets used and/or analysed during the current study are available from the corresponding author on reasonable request. 


\section{Conflict of interest}

The following patents are relevant to the work in this manuscript.

- PCT/EP2019/087056. L-triiodothyronine (T3) for use in limiting microvascular obstruction.

- Greek Patent Office, number of case: 22-0002577373. Composition comprising L-triiodothyronine (T3) for use in the treatment of critically ill patients with coronavirus infection.

- PCT/4972/2021. Pharmaceutical composition comprising L-triiodothyronine (T3) for use in the treatment of tissue hypoxia and sepsis (pending)

$\mathrm{CP}$ and IM are the inventors and hold royalties in relation to these patents.

\section{Funding}

This project is supported by a grant from Uni-Pharma Pharmaceutical Company. The sponsor has no involvementin the design of the study, collection, analysis, and interpretation of data and in writing the manuscript. The study is implemented by a CRO company (PHARMASSIST).

\section{Acknowledgments}

We would like to thank Prof. Georgia Kostopanagiotou, Dr. A. Alexandrou, Dr. M. Avdikou, Dr. M. Giannopoulou and Dr. A. Vassi, for their valuable support in the study.

\section{References}

[1] Mourouzis IS, Lourbopoulos AI, Trikas AG, Tseti IK, Pantos CI. Triiodothyronine prevents tissue hypoxia in experimental sepsis: potential therapeutic implications. Intensive Care Med Exp. 2021;9(1):17.

[2] Pantos C, Mourouzis I, Saranteas T, Clavé G, Ligeret H, Noack-Fraissignes P, et al.Thyroid hormone improves postischaemic recovery of function while limiting apoptosis: a new therapeutic approach to support hemodynamics in the setting of ischaemia-reperfusion? Basic Res Cardiol. 2009;104(1):69-77.

[3] Pantos C, Tseti I, and Mourouzis I. Use of triiodothyronine to treat critically ill COVID-19 patients: a new clinical trial. Crit Care. 2020;24:209.

[4] Pantos C, Kostopanagiotou, G, Armaganidis A, Trikas A, Tseti I, Mourouzis I. Triiodothyronine for the treatment of critically ill patients with COVID-19 infection: A structured summary of a study protocol for a randomised controlled trial. Trials. 2020;21:573.

[5] Dadgostar H, Holland GN, Huang X, Tufail A, Kim A, Fisher TC, et al. Hemorheologic abnormalities associated with HIV infection: in vivo assessment of retinal microvascular blood flow. Invest Ophthalmol Vis Sci. 2006;47(9):3933-8.

[6] Yang Y, Che Y, Yang L. Relationship of serum inflammatory cytokines with anemia and vascular endothelial function in children with systemic lupus erythematosus. Clin Hemorheol Microcirc. 2019;73(2):299-306. doi: 10.3233/CH-180492.

[7] Farber PL. Can erythrocytes behavior in microcirculation help the understanding the physiopathology and improve prevention and treatment for covid-19? Clin Hemorheol Microcirc. 2021 (in press). doi: 10.3233/CH-201082.

[8] Martini R. The compelling arguments for the need of microvascular investigation in COVID-19 critical patients. Clin Hemorheol Microcirc. 2020;75(1):27-34. doi: 10.3233/CH-200895.

[9] Saldanha C. Erythrocyte a Target for Covid-19 Infected Patients. Mod Appro Drug Des. 3(1).MADD.000555.2020.

[10] Renoux C, Fort R, Nader E, Boisson C, Joly P, Stauffer E, et al. Impact of COVID-19 on red blood cell rheology. Br J Haematol. 2021;192(4):e108-e111.

[11] Lapić I, Rogić D, Plebani M. Erythrocyte sedimentation rate is associated with severe coronavirus disease 2019(COVID19): a pooled analysis. Clin Chem Lab Med. 2020;58(7):1146-8.

[12] Ackermann M, Verleden SE, Kuehnel M, Haverich A, Welte T, Laenger F, et al. Pulmonary Vascular Endothelialitis, Thrombosis, and Angiogenesis in Covid-19. N Engl J Med. 2020;383(2):120-8. 